\title{
Stent-induced coronary artery stenosis characterized by multimodal nonlinear optical microscopy
}

\author{
Han-Wei Wang, ${ }^{a}$ Vlad Simianu, ${ }^{b}$ Mattew J. Locker, ${ }^{b}$ Ji-Xin Cheng, ${ }^{a, c}$ and Michael Sturek ${ }^{a, b}$ \\ a Purdue University, Weldon School of Biomedical Engineering, West Lafayette, Indiana 47907 \\ ${ }^{b}$ Indiana University Medical School, Department of Cellular \& Integrative Physiology, Indianapolis, Indiana 46202 \\ ${ }^{\mathrm{c}}$ Purdue University, Department of Chemistry, West Lafayette, Indiana 47907
}

\begin{abstract}
We demonstrate for the first time the applicability of multimodal nonlinear optical (NLO) microscopy to the interrogation of stented coronary arteries under different diet and stent deployment conditions. Bare metal stents and Taxus drug-eluting stents (DES) were placed in coronary arteries of Ossabaw pigs of control and atherogenic diet groups. Multimodal NLO imaging was performed to inspect changes in arterial structures and compositions after stenting. Sum frequency generation, one of the multimodalities, was used for the quantitative analysis of collagen content in the peristent and in-stent artery segments of both pig groups. Atherogenic diet increased lipid and collagen in peristent segments. In-stent segments showed decreased collagen expression in neointima compared to media. Deployment of DES in atheromatous arteries inhibited collagen expression in the arterial media. 02011 Society of Photo-Optical Instrumentation Engineers (SPIE). [DOI: 10.1117/1.3533313]
\end{abstract}

Keywords: multimodal nonlinear optical microscopy; stenosis, coronary arteries; Ossabaw miniature swine; drug eluting stents. Paper 10302SSR received Jun. 2, 2010; revised manuscript received Sep. 23, 2010; accepted for publication Sep. 30, 2010; published online Feb. 2, 2011.

\section{Introduction}

Regarded as an iatrogenic disease, in-stent restenosis is a complex response after coronary stenting ${ }^{1}$ and still remains a clinical problem. ${ }^{2,3}$ Although stenting very effectively recovers lumen size acutely, there is restenosis and, despite impressive attenuation of restenosis by drug-eluting stents (DESs), longterm anticoagulation therapy is required to decrease the risk of thrombosis. ${ }^{1,3,4}$ Factors in the restenosis occurrence, also known as neointimal hyperplasia, have been debatable topics for decades ${ }^{5-7}$ Molecular expression, vessel size, stent type, eluting drug coating, stenting methods, etc., may all affect neointimal hyperplasia. ${ }^{5,6}$ It is widely accepted that smooth muscle cells are involved in neointimal hyperplasia. The inflammatory response, proliferation, and migration of smooth muscle cells, and thus its responses of extracellular matrix formation highly contribute to the process of restenosis through cellular and molecular pathophysiological pathways. ${ }^{4,7}$ Therefore, the major efforts to reduce restenosis have been placed on inhibiting the activation and migration of smooth muscle cells. ${ }^{8,9}$ Accumulation of extracellular matrices that contribute highly to in-stent restenosis has also been highlighted. ${ }^{10,11}$ To evaluate stent efficacy and composition of the vascular wall in restenosis, imaging techniques have been taking on key roles. Beyond histological evaluations that have been established as the gold standard for biopsy inspections, magnetic resonance imaging and computed tomography have been demonstrated to identify luminal narrowing after stent placement. ${ }^{12,13}$ Angiography and intravascular ultrasound have also been used not only to optimize the guidance of stent implantation, ${ }^{14,15}$ but also to quantify severity of restenosis. ${ }^{16}$

Address all correspondence to: Ji-Xin Cheng, 206 S. Martin Jischke Drive, West Lafayette, Indiana 47907-2032. Tel: 765 494-4335; Fax: 765 496-1912; E-mail: jcheng@purdue.edu, Michael Sturek, 635 Barnhill Drive, Room 385, Indianapolis, IN 46202-5120, Tel: 317 274-7772; Fax: 317 274-3318; email: msturek@iupui.edu.
Although intravascular ultrasound can provide some identification of arterial wall composition, ${ }^{12}$ only histological staining provides cellular details and compositional specificity.

There has been an increasing focus in the past decade on using nonlinear optical (NLO) microscopy ${ }^{17}$ for imaging biological tissues because of its unique advantages, such as inherent 3-D resolution and near-infrared (NIR) excitation for superior optical penetration. ${ }^{18}$ Notably, by utilizing intrinsic sources of NLO signals, NLO imaging has been used intensively for studies of unstained tissues. ${ }^{19-27}$ For vascular studies, elastin and collagen fibrils can be visualized without labeling by twophoton excitation fluorescence (TPEF) and second harmonic generation (SHG) microscopies, respectively. ${ }^{22,28}$ Recently, an integration of multiple NLO modalities has been used for the identification of vasculature and arterial cells. ${ }^{29}$ Sum frequency generation $(\mathrm{SFG})^{30}$ was used for the visualization of arterial collagen matrices. The SFG that derives its signal from noncentrosymmetric molecules at the sum frequency of two excitation sources showed imaging capability similar to that of SHG for biological tissues. ${ }^{25}$ Beyond endogenous sources of NLO signals, two-photon microscopy gave remarkable results in imaging arterial cells with the aid of labels. ${ }^{31,32}$ For atherosclerosis studies, NLO microscopy has also been demonstrated to provide insightful information. ${ }^{33-37}$ Le et al. applied coherent anti-stokes Raman scattering (CARS) microscopy to visualize lipid-laden cells in atheroma. ${ }^{33}$ Lilledahl et al. recently utilized SHG and TPEF imaging to identify the fibrous cap of vulnerable plaques. ${ }^{34}$ Our recent work further utilized a CARS-based NLO microscopy for characterizing different atherosclerotic stages from early to advanced lesions. ${ }^{35}$ Regarding restenosis, however, it is still unknown whether NLO microscopy can provide morphometric and quantitative analysis. The modalities of NLO microscopy hold great promise to be useful in the

1083-3668/2011/16(2)/021110/6/\$25.00 (C) 2011 SPIE 


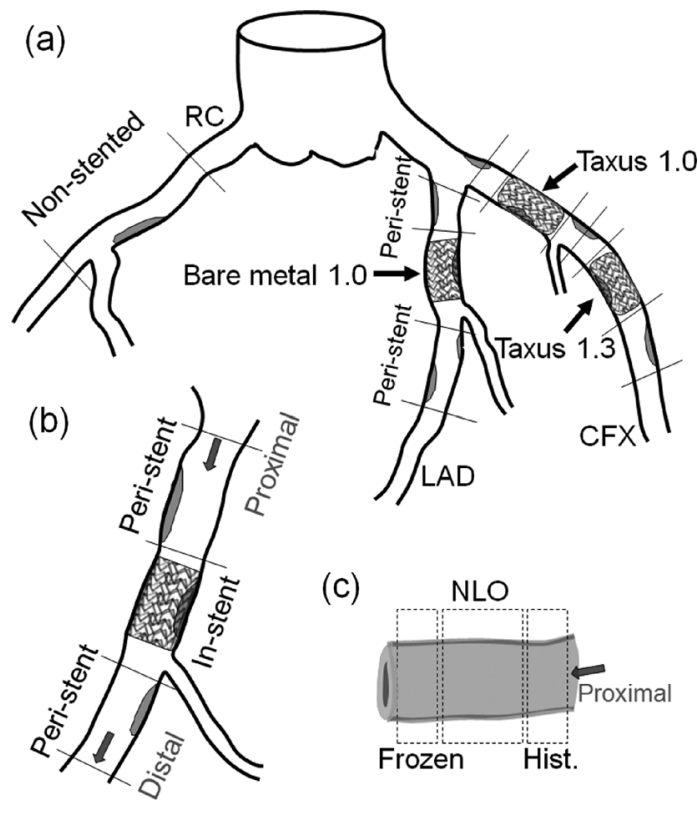

Fig. 1 Schematic of coronary stent deployment and sample collection. (a) Schematic drawing of coronary arteries and the study design. (b) Collected arterial sections included peristent and in-stent segments. (c) Subsegments collected for NLO imaging and histology. The right coronary (RC) artery had no stents deployed in it (nonstented).

characterization of restenotic arteries, which may assist in the design and improvement of stent therapy. To address these needs, in the present study we demonstrate for the first time the applicability of multimodal NLO microscopy to the interrogation of stented coronary arteries. The label-free imaging allowed visualization of the degree of in-stent stenosis and, more importantly, cellular and extracellular matrix composition. Quantitative analyses of collagen deposition in stented arteries were performed using the SFG signal. The results revealed the effects of the diet and DES placement on the collagen expression in coronary arteries of a clinically relevant porcine model.

\section{Experimental Methods}

\subsection{Experimental Design}

The experimental procedures and protocols were approved by an Institutional Animal Care and Use Committee and complied fully with Ref. 38 . Ossabaw miniature pigs $(N=10)$ were placed on diets for 22-24 weeks before stenting. Lean Ossabaw control pigs $(N=5)$ were fed with a normal maintenance diet, which contained $8 \% \mathrm{kcal}$ from fat, $22 \% \mathrm{kcal}$ from protein, $70 \% \mathrm{kcal}$ from carbohydrates. Obese, metabolic syndrome (MetS) pigs $(N=5)$ were fed a high-fat/high-cholesterol, atherogenic diet, which was composed of $2 \%$ cholesterol, $20 \%$ kcal from fructose, and $46 \% \mathrm{kcal}$ from hydrogenated soybean oil that is high in trans fatty acids. ${ }^{39}$ The genetic predisposition of Ossabaw pigs to obesity and metabolic syndrome promotes the development of cardiovascular disease ${ }^{15,16}$ and greater in-stent neointimal hyperplasia compared with other leaner breeds, such as Yucatan and domestic. ${ }^{15,16,40}$

Procedures of surgery and stent deployment can be found elsewhere. ${ }^{11,16,41}$ Three stents were deployed in the distal circumflex (CFX) and left anterior descending (LAD) coronary arteries of each pig in both groups [Fig. 1(a)]. Three stent deployments were bare metal stent (BMS) with $1.0 \times$ expansion, relative to the normal lumen diameter and Taxus stent (Boston Scientific, Inc., Natick, MA.) with $1.0 \times$ and $1.3 \times$ expansion ratios. ${ }^{15,16,40}$ The Taxus stent is a paclitaxel-coated DES. Before stent placement, intravascular ultrasound and angiographic imaging were used to measure the lumen diameter.

Coronary arteries from these two groups were harvested after four weeks of recovery from stent placement. ${ }^{15}$ A sternotomy was performed to excise the heart into cold saline. Coronary artery segments were then collected. Segments covered peristent and in-stent locations of the three kinds of stent deployments [Fig. 1(b)]. Peristent segments were derived 2-5 mm proximal and distal to the stent. Right coronary arteries, where no stents was deployed, were also collected as a control representing the natural course of healthy or atherosclerotic coronary arteries. Each segment collected was sectioned into subsegments for NLO microscopy and histology [Fig. 1(c)]. Collected arterial segments were encoded for blind imaging and analysis. Arteries were preserved by zinc formalin solution after harvest and washed with phosphate buffered saline before NLO imaging.

\subsection{Multimodal Nonlinear Optical Microscopy}

Details of our multimodal imaging setup can be found elsewhere. ${ }^{29}$ Briefly, two synchronized 5-ps lasers (Tsunami, Spectra-Physics, Irvine, CA.) operating at an $80-\mathrm{MHz}$ repetition rate were parallel-polarized, collinearly combined, and directed into a laser-scanning microscope (FV300/IX71, Olympus, Center Valley, PA.). A $20 \times$ air objective with a numerical aperture (NA) of 0.75 and a $60 \times$ water immersion objective with a $1.2 \mathrm{NA}$ were used for the NLO imaging. Mosaic images were stitched manually with subimages taken by the $20 \times$ objective. Subimages were highly overlapped with each other at $\sim 75 \%$ of the imaging area of a $353 \times 353 \mu \mathrm{m}^{2}$ field of view. Signals were detected by external photomultiplier tubes (H7422-40, Hamamatsu, Bridgewater, NJ.).

For CARS imaging, ${ }^{42}$ the frequency difference of the two NIR lasers was tuned to $2840 \mathrm{~cm}^{-1}$, which matches the symmetric $\mathrm{CH}_{2}$ stretch vibration. ${ }^{42,43}$ Bandpass filters $(600 / 65 \mathrm{~nm}$, Ealing Catalog, Rocklin, CA.) were used to transmit the CARS signal of $\sim 588 \mathrm{~nm}$. The average powers of the pump and the Stokes laser beams at the sample were 40 and $20 \mathrm{~mW}$, respectively. The same lasers were used to generate the SFG signal at $\sim 393 \mathrm{~nm}$. Two bandpass filters (HQ375/50m-2p, Chroma, Bellows Falls, VT.) were used for the collection of the backward SFG signal. For TPEF imaging of unlabeled samples, two bandpass filters (hp520/40m-2p, Chroma) were used to transmit autofluorescence. For TPEF imaging of arterial cell nuclei labeled by Doxorubicin, two 600/65-nm filters were used. The TPEF signal was generated by only using the pump laser $(\sim 707$ $\mathrm{nm}$ ) to avoid the CARS signal at $\sim 588 \mathrm{~nm}$.

\subsection{Histological Imaging}

The arterial segments for the comparison to histology were routinely processed with paraffin embedding and then sectioned for staining. Hematoxylin and eosin was used for the identification of general cell morphology, including the lipid-rich cells that give bubbly and granular appearances. Masson's trichrome was 
Table 1 In-stent and peristent arterial specimens of different stent deployments in different diet groups.

\begin{tabular}{ccccccccc}
\hline & \multicolumn{3}{c}{ Control } & & \multicolumn{4}{c}{ MetS } \\
\cline { 2 - 3 } \cline { 6 - 8 } & Peristent & In-stent & Vessel & Peristent & In-stent & Vessel \\
\hline BMS 1.0 & 4 & 5 & LAD & & 4 & 5 & LAD \\
DES 1.0 & 2 & 4 & CFX & 4 & 5 & CFX \\
DES 1.3 & 4 & 4 & CFX & 5 & 5 & CFX \\
\hline
\end{tabular}

1.0 and 1.3: Fold expansion of stent relative to normal lumen diameter.

used to identify collagen. Images were captured with a Nikon CoolPix 990 digital camera on a Nikon Diaphot inverted microscope (Nikon, Melville, NY).

\subsection{Quantitative Analysis}

Collagen content was quantitatively assessed by SFG and histology images. Serial sections of arterial samples were used for a correlation study between SFG and histological evaluations. Measurements were performed with Image Pro Plus v.4.1 (Media Cybernetics, Bethesda, MD.). For histology images, collagen content in tunica media and in adventitia of an artery was determined according to Masson's trichrome staining. The measurement was made by colorimetric analysis with the adventitia as a reference color template. Pixels presented the color of collagen stain were retrieved from the 24-bit, full-color images, and then the area percentage was derived as compared to the total pixels in the area selected. For SFG images, collagen content was measured according to the SFG signal. Area percentage of collagen was derived according to the percentage of pixels that gave a positive signal over a threshold. Background threshold, color code, and color scale were set identically for the routine analyses. Each data point in the correlation graph is an average value of 12 rectangular, $(50 \times 50)-\mu \mathrm{m}^{2}$, areas in an arterial stratification along the arterial ring. The same analysis routines were then applied to the study of SFG quantitation of collagen content. ${ }^{32}$

\subsection{Statistical Analysis}

Results are presented as mean $\pm \operatorname{SD}(p<0.01)$. The paired student $t$-test was used to identify the statistical differences among means in the correlation study of collagen analysis between SFG and histology imaging. One-way analysis of variance (ANOVA) with a posthoc testing (Tukey's test) was performed to identify the statistical differences among groups. The threshold for statistical significance was $p=0.05$.

\section{Results}

Coronary arteries deployed with the $1.0 \times$ BMS, $1.0 \times$ Taxus, and $1.3 \times$ Taxus (Table 1 ) were interrogated by the multimodal NLO microscopy. Adjacent arterial sections were histologically stained to verify the integrity of the NLO imaging. For peristent samples, 10 arterial sections from the control group and
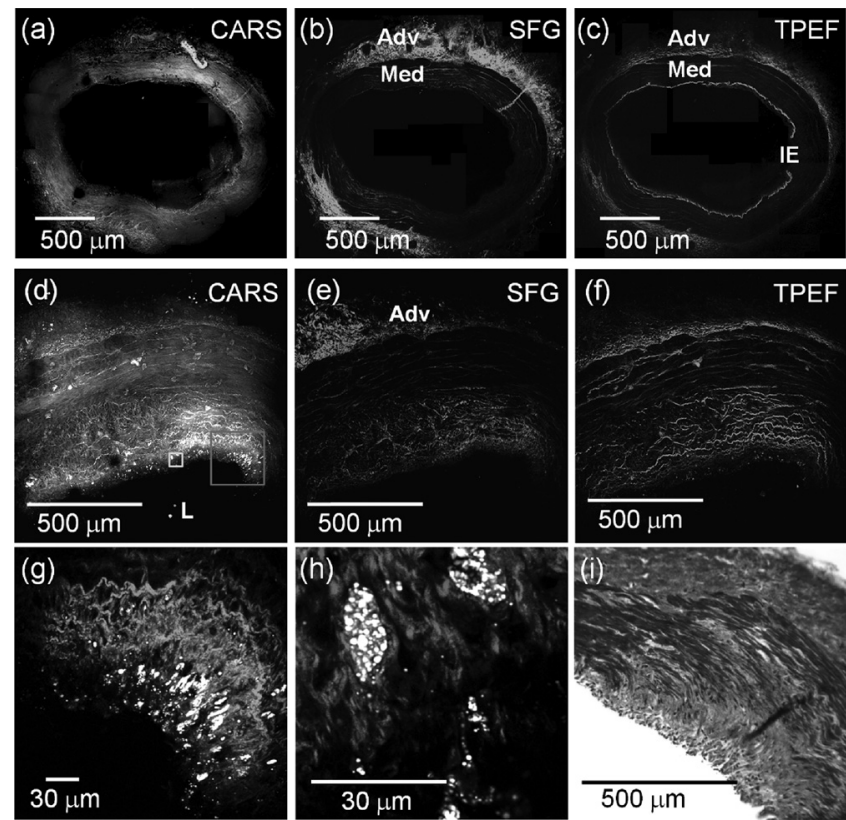

Fig. 2 (Color online only.) NLO images of peristent arteries showed typical normal and atherosclerotic composition. (a-c) are mosaic images of CARS (lipid, cell structure), SFG (collagen), and TPEF (elastin), respectively, of a peristent coronary artery segment from the control group. Normal arterial structure was preserved at the peristent segment of a $1.0 \times$ BMS deployment. Adventitia (Adv) shows typically abundance of collagen (blue) and elastin (green), while the media (Med) has little collagen and elastin. The internal elastic (IE) lamina is distinct in healthy arteries. Each mosaic image contains 35 subimages. $(d-f)$ are images of an early stage atheroma observed by CARS, SFG, and TPEF, respectively, in a peristent sample in the atherogenic diet MetS group. The mosaic images, formed with 36 subimages, at the peristent location show characteristics of an early stage atheroma that included a scattered lipid accumulation, higher collagen expression, and disorganized elastic lamellae in thickened intima and adjacent media. (g) A colocalized image of three NLO modalities at the red box in (c) shows the scattered lipid deposits (white) in the thickened intima with interlacing of collagen and elastin. (h) Zoomed-in image at the yellow box in (d) shows the pathologic infiltration of lipid-rich cells and corresponding collagen matrices. (i) Histological imaging of Masson's trichrome at corresponding segment showing collagen (blue) and cellular material in the media (red), while a predominance of collagen in the adventitia. L: Lumen. CARS: gray, SFG: blue, TPEF: green. The same color code is used in other images.

13 from the metabolic syndrome group were imaged. Figure 2 shows representative images of arterial structures visualized in peristent samples from both groups. Typically, peristent samples from the control group showed normal arterial vasculature [Figs. 2(a)-2(c)] and gave no observable difference from the structures in right coronary control samples. Organized lamellae of elastin and collagen were clearly identified in tunica media. By contrast, atheroma [Figs. 2(d)-2(f)], characterized by scattered lipid accumulations [Figs. 2(g) and 2(h)] and a thickened intima [Figs. 2(d)-2(f) and 2(i)], was frequently observed in the peristent coronary arteries of the MetS group. In general, arterial structures and lesions were well protected at the peristent locations from the mechanical disturbance of stent deployment.

To interrogate in-stent arterial segments, a total sample number of 13 of the three deployment conditions in the control group and 15 of those in the high-fat group were imaged. Figure 3 exemplifies the arterial composition in a 1.0 Taxus 

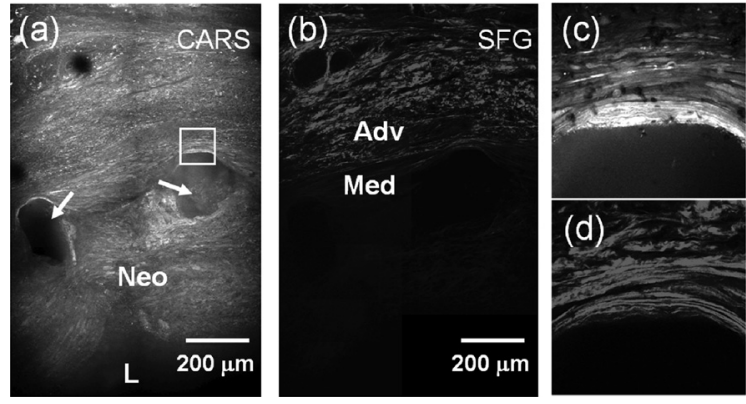

Fig. 3 Atrophy in media and low collagen expression in stenotic tissue are typical in arteries with 1.0x Taxus stents. The mosaic image of CARS in (a) shows the locations of struts (arrows) and the stenotic tissue (neointimal hyperplasia) under it. The SFG image in (b) detects the distribution of collagen in the stented arteries without labels and shows low collagen in the media and neointima. (c,d) are zoomed-in images of CARS and SFG, respectively, at the box in (a). Strong CARS and SFG signals were observed above the strut location. Straightened collagen fibrils were observed along the strut locations. Neo: neointimal hyperplasia.

deployed coronary artery of the MetS group. Locations of stent struts identified in CARS and SFG images [Figs. 3(a) and 3(b)] allowed circumscribing the area of in-stent neointimal hyperplasia. In-stent artery segments can thus be identified due to the cellular contrast in the CARS images. Around the strut locations, the edges close to the arterial wall showed higher CARS and SFG signals [Figs. 3(c) and 3(d)], thus implying a lipid accumulation and abundant collagen deposition. Higher lipid content at these locations is likely due to the compression of the stents to the prestent lipid accumulation at the lumen area. A higher collagen signal at the edges of the stent strut could result from the compressed collagen layers and possibly the activation of collagen synthesis along with the mechanical pressure exerted by the stents. ${ }^{44}$ In addition, atrophy of media [Figs. 3(a)-3(b)] was a typical occurrence at the strut locations.

Compared to 1.0-Taxus-deployed coronaries, images of 1.3Taxus-deployed coronaries showed greater atrophy at strut locations. Figure 4 shows a representative image of a coronary artery deployed with a 1.3 Taxus. The absence of arterial layers above strut locations [Figs. 4(a) and 4(b)] shows further
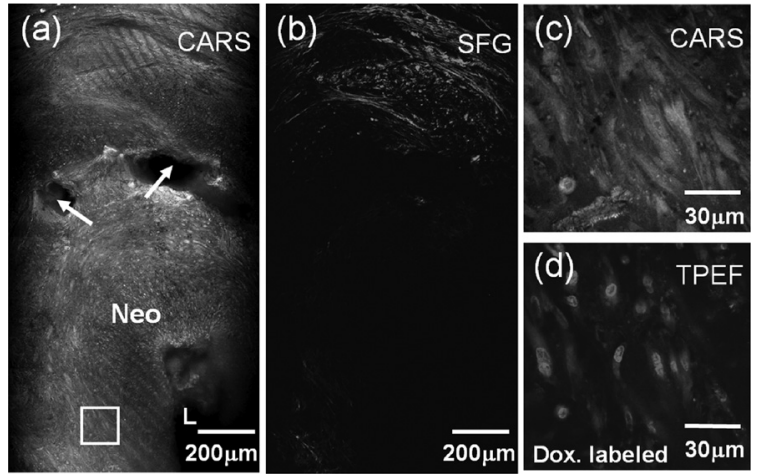

Fig. 4 Representative images of a $1.3 \times$ Taxus stent. Minimal media was observed in (a) CARS and (b) SFG images. (c) CARS image taken at the rectangle in (a) shows the proliferating stellate-shaped cells in the stenotic tissue. (d) Cell nuclei were confirmed by doxorubicin (Dox.) labeling.
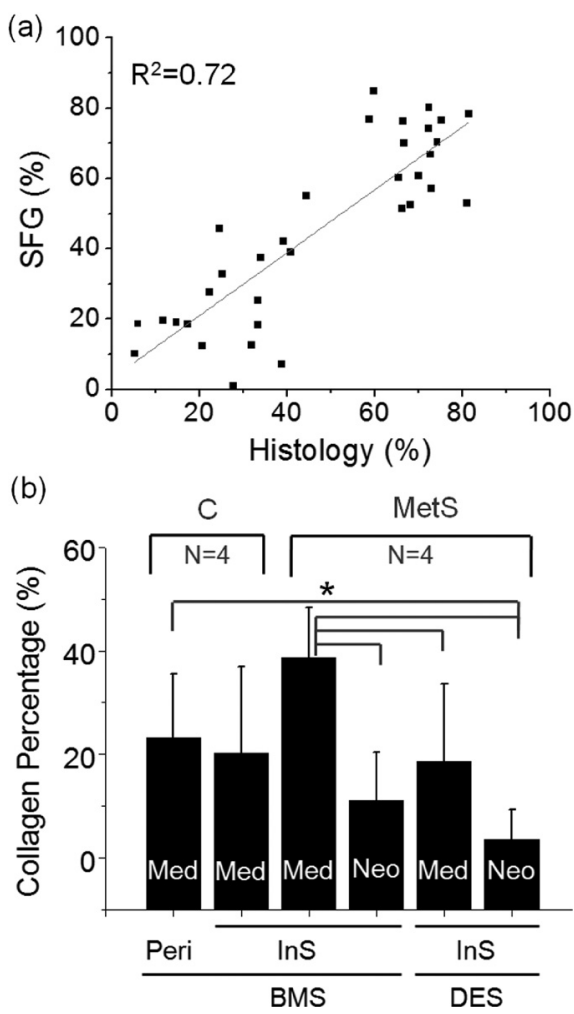

Fig. 5 Quantitative analysis of collagen according to the SFG signal in stented arteries. (a) Correlation of collagen percentage between the SFG and histological evaluations $\left(R^{2}=0.72\right)$. (b) Comparison of collagen percentages among different locations of different groups with different stents. C: control group; MetS: metabolic syndrome group; BMS: Bare metal stent of 1.0 expansion ratio; DES: Taxus drug-eluting stent of 1.0 expansion ratio; Peri: peristent sample; InS: in-stent sample; Med: media; Neo: neointimal hyperplasia. The asterisk and bars indicate the significant differences between specific groups $(P<0.05)$.

damage of the arterial wall, most likely attributed to the overexpansion of the stent. Moreover, NLO imaging gave clear images of stenotic arteries due to the subcellular imaging resolution and showed a predominantly cellular composition [Figs. 4(c) and 4(d)]. Stellate-shaped cells were observed in the stenotic arteries by the CARS imaging. Collagen content in the stenoses (neointima), however, was lower than that in the arterial wall peripheral to the stent.

For the analysis of the collagen expression in the stented coronaries, SFG images of the stented coronaries were quantitatively analyzed (Fig. 5). The correlation study shows that the SFG signal gives a valid collagen quantity by comparison to the morphometric results of histological staining [Fig. 5(a)]. Comparing the collagen expression in the media between the stented and peristent samples of BMS-deployed arteries in the control group, there was no significant difference observed [Fig. 5(b)]. The result shows that the deployment of the stent at normal lumen diameter did not promote collagen expression in the media. In contrast, the atherogenic diet MetS group showed a trend for higher collagen content in the media [Fig. 5(b)], but a significant difference was not found using one-way ANOVA with the posthoc testing. The trend for higher collagen content may imply that the activation of smooth muscle cell synthetic phenotype in the media was enhanced by the atherogenic stimulus. The major result was a lower collagen content in the neointima compared 
to media, and the result was in keeping with former reports on Ossabaw swine. ${ }^{45}$ Interestingly, there is a significant difference in the collagen content of stenotic arteries between BMS- and DES-deployed arteries. The collagen content was lower in both neointima and media with the deployment of drug eluting stents [Fig. 5(b)]. Although there was not a significant difference between the collagen in neointima of the BMS versus DES groups, there was decreased collagen in the media of the DES compared to BMS group.

\section{Discussion}

In the present study, we interrogated in-stent and peristent coronary arterial samples by multimodal NLO microscopy. The major finding is that we have shown with label-free NLO imaging cellular proliferation, lipid accumulation, and extracellular matrix changes in the arterial media and neointima after stent deployment. Quantification of collagen in neointima was correlated with "gold-standard" histological methods. The discrepancy in the analysis of collagen is most likely because of the variation between serial sections used in NLO and histological analyses. Nevertheless, the correlation provided confidence in our finding that, compared to bare metal stents, paclitaxel-eluting stents decrease collagen extracellular matrix production in arterial media and possibly neointima.

Mosaic imaging was carried out to provide a large area of inspection qualitatively and quantitatively. Stellate-shaped cells were visualized in CARS images in the classical neointimal hyperplasia in the stenotic, in-stent artery segments. There was a clear predomination of proliferating smooth muscle cells in the areas. ${ }^{46-48}$ Meanwhile, SFG analysis showed that neointima collagen content was lower than that in the arterial media and even lower in DES-deployed in-stent segments. The result suggested that DES deployment inhibits collagen extracellular matrix production by synthetic phenotype smooth muscle cells, which may allow a great accumulation of fibrin. ${ }^{48}$ It is known that the increase of extracellular matrix provides the bulk of the in-stent neointimal hyperplasia; ${ }^{11,47,48}$ therefore, the inhibition of collagen expression by the DES is a major factor in preventing restenosis. Although it is well established that paclitaxel DES inhibits smooth muscle cell proliferation, as well as extracellular matrix formation in vitro and clinically, ${ }^{47-51}$ the influences of the predisposition to neointimal hyperplasia and the drugrelease profile could play critical roles. Moreover, the collagen content in the media of DES-deployed arteries is lower than that in the media of BMS-deployed arteries in the MetS group (Fig. 5), thus indicating that drug released from the stent reached the tunica media. ${ }^{52,53}$ Furthermore, the data suggest that DES may inhibit the shift of contractile smooth muscle cells in the media to a synthetic phenotype that predominates in the neointima.

It is notable that instead of stains used in histopathology, cellular characterization was derived by CARS microscopy without labels. Along with the collagen evaluation by SFG imaging, the NLO multimodality provided virtual histology for the identification of cellular structures and collagen fibrils. Additional labels that can be visualized by TPEF modality can further extend the horizons of compositional imaging in vascular pathology. The quantitative analysis in this work demonstrated the applicability of multimodal NLO microscopy to the interrogation of stented coronary arteries. The SFG signal used for quantifying collagen content showed the effects of stent deployment. More extensive application of multimodal NLO, including coherent Raman scattering processes without exogenous labels, to characterize in-stent restenosis and drug effects, may accelerate studies of DES design and selection and therapeutic intervention.

\section{Acknowledgments}

This work was supported by a NIH R01 Grant No. EB007243 to J. X. C., NIH Grant Nos. RR013223 and HL062552 to M.S., and the Comparative Medicine Program of Purdue and Indiana University School of Medicine and the Summer Research Program in Academic Medicine program of Indiana University School of Medicine. We thank Dr. Eric A. Mokelke of Boston Scientific, Inc., for the gift of Taxus stents.

\section{References}

1. N. Kukreja, Y. Onuma, J. Daemen, and P. W. Serruys, "The future of drug-eluting stents," Pharmacol. Res. 57(3), 171-180 (2008).

2. Y. Li, Z. Zheng, B. Xu, S. Zhang, W. Li, R. Gao, and S. Hu, "Comparison of drug-eluting stents and coronary artery bypass surgery for the treatment of multivessel coronary disease: Three-year follow-up results from a single institution," Circulation 119(15), 2040-2050 (2009).

3. A. Sourgounis, J. Lipiecki, T. S. Lo, and M. Hamon, "Coronary stents and chronic anticoagulation," Circulation 119(12), 1682-1688 (2009).

4. A. K. Mitra and D. K. Agrawal, "In stent restenosis: bane of the stent era," J. Clin. Pathol. 59(3), 232-239 (2006).

5. C. Bauters and J. M. Isner, "The biology of restenosis," Prog. Cardiovasc. Dis. 40(2), 107-116 (1997).

6. A. Kastrati, A. Dibra, J. Mehilli, S. Mayer, S. Pinieck, J. Pache, J. Dirschinger, and A. Schomig, "Predictive factors of restenosis after coronary implantation of sirolimus- or paclitaxel-eluting stents," Circulation 113(19), 2293-2300 (2006).

7. V. Rajagopal and S. G. Rockson, "Coronary restenosis: a review of mechanisms and management," Am. J. Med. 115(7), 547-553 (2003).

8. M. N. Babapulle and M. J. Eisenberg, "Coated stents for the prevention of restenosis: Part I," Circulation 106(21), 2734-2740 (2002).

9. D. E. Drachman, E. R. Edelman, P. Seifert, A. R. Groothuis, D. A. Bornstein, K. R. Kamath, M. Palasis, D. Yang, S. H. Nott, and C. Rogers, "Neointimal thickening after stent delivery of paclitaxel: change in composition and arrest of growth over six months," J. Am. Coll. Cardiol. 36(7), 2325-2332 (2000).

10. D. D. Allison, T. N. Wight, N. J. Ripp, K. R. Braun, and K. J. GrandeAllen, "Endogenous overexpression of hyaluronan synthases within dynamically cultured collagen gels: Implications for vascular and valvular disease," Biomaterials 29(20), 2969-2976 (2008).

11. I.-M. Chung, H. K. Gold, S. M. Schwartz, Y. Ikari, M. A. Reidy, and T. N. Wight, "Enhanced extracellular matrix accumulation in restenosis of coronary arteries after stent deployment," J. Am. Coll. Cardiol. 40(12), 2072-2081 (2002).

12. D. A. Bluemke, S. Achenbach, M. Budoff, T. C. Gerber, B. Gersh, L. D. Hillis, W. G. Hundley, W. J. Manning, B. F. Printz, M. Stuber, and P. K. Woodard, "Noninvasive coronary artery imaging: Magnetic resonance angiography and multidetector computed tomography angiography: a scientific statement from the american heart association committee on cardiovascular imaging and intervention of the council on cardiovascular radiology and intervention, and the councils on clinical cardiology and cardiovascular disease in the young," Circulation 118(5), 586-606 (2008).

13. W. G. Hundley, L. D. Hillis, C. A. Hamilton, R. J. Applegate, D. M. Herrington, G. D. Clarke, G. A. Braden, M. S. Thomas, R. A. Lange, R. M. Peshock, and K. M. Link, "Assessment of coronary arterial restenosis with phase-contrast magnetic resonance imaging measurements of coronary flow reserve," Circulation 101(20), 2375-2381 (2000).

14. P. Roy, D. H. Steinberg, S. J. Sushinsky, T. Okabe, T. L. P. Slottow, K. Kaneshige, Z. Xue, L. F. Satler, K. M. Kent, W. O. Suddath, A. D. 
Pichard, N. J. Weissman, J. Lindsay, and R. Waksman, "The potential clinical utility of intravascular ultrasound guidance in patients undergoing percutaneous coronary intervention with drug-eluting stents," Eur. Heart. J. 29, 1851-1857 (2008).

15. M. Sturek, M. Alloosh, J. Wenzel, J. P. Byrd, J. M. Edwards, P. G. Lloyd, J. D. Tune, K. L. March, M. A. Miller, E. A. Mokelke, and I. L. Brisbin, "Ossabaw island miniature swine: Cardiometabolic syndrome assessment," Chapter 18 in Swine in the Laboratory: Surgery, Anesthesia, Imaging, and Experimental Techniques, M. M. Swindle, Ed., CRC Press, Boca Raton (2007).

16. J. M. Edwards, M. Alloosh, X. Long, G. M. Dick, P. G. Lloyd, E. A. Mokelke, and M. Sturek, "Adenosine A1 receptors in neointimal hyperplasia and in-stent stenosis in Ossabaw miniature swine," Coron. Artery Dis. 19(1), 27-31 (2008).

17. W. R. Zipfel, R. M. Williams, and W. W. Webb, "Nonlinear magic: multiphoton microscopy in the biosciences," Nat. Biotech. 21, 13691377 (2003).

18. F. Helmchen and W. Denk, "Deep tissue two-photon microscopy," Nat. Meth. 2(12), 932-940 (2005).

19. L. Moreaux, O. Sandre, and J. Mertz, "Membrane imaging by secondharmonic generation microscopy," J. Opt. Soc. Am. B 17, 1685-1694 (2000).

20. A. Zoumi, A. Yeh, and B. J. Tromberg, "Imaging cells and extracellular matrix in vivo by using SHG and TPEF.," Proc. Natl. Acad. Sci. USA 99 11014-11019 (2002).

21. P. J. Campagnola and L. M. Loew, "Second-harmonic imaging microscopy for visualizing biomolecular arrays in cells, tissues and organisms," Nat. Biotech. 21, 1356-1360 (2003).

22. W. R. Zipfel, R. M. Williams, R. Christie, A. Y. Nikitin, B. T. Hyman, and W. W. Webb, "Live tissue intrinsic emission microscopy using multiphoton-excited native fluorescence and second harmonic generation," Proc. Natl. Acad. Sci. USA 100, 7075-7080 (2003).

23. C. L. Evans, E. O. Potma, M. Puoris'haag, D. Côté, C. P. Lin, and X. S. Xie, "Chemical imaging of tissue in vivo with video-rate coherent anti-Stokes Raman scattering microscopy," Proc. Natl. Acad. Sci. U.S.A. 102(46), 16807-16812 (2005).

24. R. M. Williams, W. R. Zipfel, and W. W. Webb, "Interpreting SHG images of collagen I fibrils," Biophys. J. 88, 1377-1386 (2005).

25. Y. Fu, H. Wang, R. Shi, and J. X. Cheng, "Second harmonic and sum frequency generation imaging of fibrous astroglial filaments in ex vivo spinal tissues," Biophys. J. 92, 3251-3259 (2007).

26. T. Hellerer, C. Axang, C. Brackmann, P. Hillertz, M. Pilon, and A. Enejder, "Monitoring of lipid storage in Caenorhabditis elegans using coherent anti-Stokes Raman scattering (CARS) microscopy," Proc. Natl. Acad. Sci. U.S.A. 104(37), 14658-14663 (2007).

27. C. W. Freudiger, W. Min, B. G. Saar, S. Lu, G. R. Holtom, C. He, J. C. Tsai, J. X. Kang, and X. S. Xie, "Label-free biomedical imaging with high sensitivity by stimulated raman scattering microscopy," Science 322(5909), 1857-1861 (2008).

28. A. Zoumi, X. Lu, G. S. Kassab, and B. J. Tromberg, "Imging coronary artery microstructure using SHG and TPEF," Biophys. J. 87, 2778-2786 (2004).

29. H. W. Wang, T. T. Le, and J. X. Cheng, "Label-free imaging of arterial cells and extracellular matrix using a multimodal nonlinear optical microscope," Opt. Commun. 281(7), 1813-1822 (2008).

30. Y. R. Shen, "Surface properties probed by SHG and SFG," Nature 337, 519-525 (1989).

31. M. Zandvoort, W. Engels, K. Douma, L. Beckers, M. Egbrink, M. Daemen, and D. W. Slaaf, "TP microscopy for imaging of the vascular wall: a proof of concept study.," J. Vasc. Res. 41, 54-63 (2004).

32. K. Douma, R. T. A. Megens, S. Reitsma, L. Prinzen, D. W. Slaaf, and M. A. M. J. V. Zandvoort, "Two-photon lifetime imaging of fluorescent probes in intact blood vessels: A window to sub-cellular structural information and binding status," Microsc. Res. Tech. 70(5), 467-475 (2007).

33. T. T. Le, I. M. Langohr, M. J. Locker, M. Sturek, and J. X. Cheng, "Label-free molecular imaging of atherosclerotic lesions using multimodal nonlinear optical microscopy," J. Biomed. Opt. 12(5), 054007 (2007).

34. M. B. Lilledahl, O. A. Haugen, C. d.L. Davies, and L. O. Svaasand, "Characterization of vulnerable plaques by multiphoton microscopy," J. Biomed. Opt. 12(4), 044005 (2007).
35. H. W. Wang, I. M. Langohr, M. Sturek, and J.-X. Cheng, "Imaging and quantitative analysis of atherosclerotic lesions by CARS-based multimodal nonlinear optical microscopy," Arterioscler. Thromb. Vasc. Biol. 29(9), 1342-1348 (2009)

36. R. S. Lim, A. Kratzer, N. P. Barry, S. Miyazaki-Anzai, M. Miyazaki, W. W. Mantulin, M. Levi, E. O. Potma, and B. J. Tromberg, "Multimodal CARS microscopy determination of the impact of diet on macrophage infiltration and lipid accumulation on plaque formation in ApoE-deficient mice," J. Lipid Res. 51(7), 1729-1737 (2010).

37. S.-H. Kim, E.-S. Lee, J. Y. Lee, E. S. Lee, B.-S. Lee, J. E. Park, and D. W. Moon, "Multiplex coherent anti-Stokes Raman spectroscopy images intact atheromatous lesions and concomitantly identifies distinct chemical profiles of atherosclerotic lipids," Circ. Res. 106(8), 1332-1341 (2010).

38. J. C. Garber, R. W. Barbee, J. T. Bielitzki, L. A. Clayton, J. C. Donovan, D. F. Kohn, N. S. Lipman, P. A. Locke, J. Melcher, F. W. Quimby, P. V. Turner, G. A. Wood, H. Würbel, L. Anestidou, Guide for the Care and Use of Laboratory Animals, National Academy Press, Washington, DC (2010).

39. I. N. Bratz, G. M. Dick, J. D. Tune, J. M. Edwards, Z. P. Neeb, U. D. Dincer, and M. Sturek, "Impaired capsaicin-induced relaxation of coronary arteries in a porcine model of the metabolic syndrome," Am. J. Physiol. Heart Circ. Physiol. 294(6), H2489-2496 (2008).

40. M. Dyson, M. Alloosh, J. Vuchetich, E. Mokelke, and M. Sturek, "Components of metabolic syndrome and coronary artery disease in female Ossabaw swine fed excess atherogenic diet," Comp. Med. 56, 35-45 (2006).

41. P. G. Lloyd, A. J. Sheehy, J. M. Edwards, E. A. Mokelke, and M. Sturek, "Leukemia inhibitory factor is upregulated in coronary arteries of Ossabaw miniature swine after stent placement," Coron. Artery Dis. 19(4), 217-226 (2008).

42. J. X. Cheng and X. S. Xie, "Coherent anti-Stokes Raman scattering microscopy: instrumentation, theory, and applications," J. Phys. Chem. B. 108, 827-840 (2004)

43. H. Wang, Y. Fu, P. Zickmund, R. Shi, and J. X. Cheng, "Coherent antistokes Raman scattering imaging of axonl myelin in live spinal tissues," Biophys. J. 89 581-591 (2005).

44. D. Hasdai, D. R. Holmes, K. N. Garratt, W. D. Edwards, and A. Lerman, "Mechanical pressure and stretch release endothelin-1 from human atherosclerotic coronary arteries in vivo," Circulation 95(2), 357-362 (1997).

45. M. Sturek, E. A. Mokelke, J. P. Vuchetich, J. M. Edwards, M. Alloosh, and K. L. March, "In-stent neointimal hyperplasia is less fibrous in metabolic syndrome Ossabaw compared to lean Yucatan swine," FASEB J. 20(5), A1399-1400 (2006).

46. R. Komatsu, M. Ueda, T. Naruko, A. Kojima, and A. E. Becker, "Neointimal tissue response at sites of coronary stenting in humans: Macroscopic, histological, and immunohistochemical analyses," Circulation 98(3), 224-233 (1998).

47. A. V. Finn, G. Nakazawa, M. Joner, F. D. Kolodgie, E. K. Mont, H. K. Gold, and R. Virmani, "Vascular Responses to Drug Eluting Stents: Importance of Delayed Healing," Arterioscler. Thromb. Vasc. Biol. 27(7), 1500-1510 (2007).

48. G. Nakazawa, A. Finn, and R. Virmani, "Vascular Pathology of DrugEluting Stents," Herz 32(4), 274-280 (2007).

49. J. Wiskirchen, W. Schöber, N. Schart, R. Kehlbach, A. Wersebe, G. Tepe, C. D. Claussen, and S. H. Duda, "The effects of paclitaxel on the three phases of restenosis: smooth muscle cell proliferation, migration, and matrix formation: an in vitro study," Invest. Radiol. 39(9), 565-571 (2004)

50. J. J. Nawarskas and L. A. Osborn, "Paclitaxel-eluting stents in coronary artery disease," Am. J. Health Syst. Pharm. 62(21), 2241-2251 (2005).

51. M. Joner, A. V. Finn, A. Farb, E. K. Mont, F. D. Kolodgie, E. Ladich, R. Kutys, K. Skorija, H. K. Gold, and R. Virmani, "Pathology of drugeluting stents in humans: delayed healing and late thrombotic risk," J. Am. Coll. Cardiol. 48(1), 193-202 (2006).

52. C. J. Creel, M. A. Lovich, and E. R. Edelman, "Arterial paclitaxel distribution and deposition," Circ. Res. 86(8), 879-884 (2000).

53. C.-W. Hwang, D. Wu, and E. R. Edelman, "Impact of transport and drug properties on the local pharmacology of drug-eluting stents," Acute Cardiac. Care 5(1), 7-12 (2003). 